\title{
473.
}

\section{ON THE GRAPHICAL CONSTRUCTION OF THE UMBRAL OR PENUMBRAL CURVE AT ANY INSTANT DURING A SOLAR ECLIPSE.}

[From the Monthly Notices of the Royal Astronomical Society, vol. xxx. (1869-1870), pp. 162-164.]

The curve in question, say the penumbral curve, is the intersection of a sphere by a right cone,-I wish to show that the stereographic projection of this curve may be constructed as the envelope of a variable circle, having its centre on a given conic, and cutting at right angles a fixed circle; this fixed circle being in fact the projection of the circle which is the section of the sphere by the plane through the centre and the axis of the cone, or say by the axial plane. The construction thus arrived at is $\mathrm{Mr}$ Casey's construction for a bicircular quartic; and it would not be difficult to show that the stereographic projection of the penumbral curve is in fact a bicircular quartic.

The construction depends on the remark that a right cone is the envelope of a variable sphere, having its centre on a given line and its radius proportional to the distance of the centre from a given point on this line; and on the following theorem of plane geometry:

Imagine a fixed circle, and a variable circle having its centre on a given line and its radius proportional to the distance of the centre from a given point on the line (or, what is the same thing, the variable circle always touches a given line); then the locus of the pole in regard to the fixed circle, of the common chord of the two circles (or, what is the same thing, the locus of the centre of a new variable circle which cuts the fixed circle at right angles in the points where it is met by the first-mentioned variable circle) is a conic.

To fix the ideas, say that $P$ is the centre of the first variable circle; $A B$ its common chord with the fixed circle; $Q$ the centre of the circle which cuts the fixed circle at right angles in the points $A$ and $B$; then the locus of $Q$ is a conic. 
To prove this, take $x^{2}+y^{2}=1$ for the equation of the fixed circle, $(x-\alpha)^{2}+(y-\beta)^{2}=\gamma^{2}$ for that of the variable circle; the foregoing law of variation being in fact such that $\alpha, \beta, \gamma$, are linear functions of a variable parameter $\theta$; the equation of the common chord $A B$ is $-2 \alpha x-2 \beta y+1+\alpha^{2}+\beta^{2}-\gamma^{2}=0$; viz., this equation contains $\theta$ quadratically; hence the envelope of the common chord is a conic; and thence (reciprocating in regard to the fixed circle) the locus of the pole of $A B$, that is, of the point $Q$, is also a conic.

Consider now a solid figure in which the circles are replaced by spheres; viz. we have a fixed sphere, and a variable sphere having its centre on a given line and its radius proportional to the distance of the centre from a given point on the line. The envelope of the variable sphere is a right cone; the intersection of the cone with the fixed sphere is the envelope of the small circle of the sphere, say the circle $A B$, which is the intersection of the fixed sphere by the variable sphere. This circle $A B$ is also the intersection of the fixed sphere by a sphere, centre $Q$, which cuts the fixed sphere at right angles; and by what precedes the locus of $Q$ is a conic. Hence the penumbral curve is given as the envelope of the circle $A B$ which is the intersection of the fixed sphere by a sphere which has its centre $Q$ on a conic, and which cuts the fixed sphere at right angles. It is obvious that the circle $A B$ always cuts at right angles the great circle which is the section of the fixed sphere by the axial plane, or say the axial circle. Project the whole figure stereographically; the projection of the circle $A B$ is a variable circle which cuts at right angles the circle which is the projection of the axial circle, and which has for its centre the point $Q^{\prime}$ which is the projection of $Q$. But the locus of $Q$ being a conic, the locus of its projection $Q^{\prime}$ is also a conic; and we have thus the projection of the penumbral curve as the envelope of a variable circle which has its centre on a conic, and which cuts at right angles a fixed circle.

We may in the axial plane construct five points of the conic which is the locus of $Q$, by means of any five assumed positions of the variable circle, and somewhat simplify the construction by a proper choice of the five positions of the variable circle. This is not a convenient construction, and even if it were accomplished we should still have to construct the projection of the conic so obtained, in order to find, in the figure of the stereographic projection, the conic which is the locus of $Q^{\prime}$. I do not at present perceive any direct construction for the last-mentioned conic; but assuming that a tolerably simple construction can be obtained, the construction of the projection of the penumbral curve as the envelope of the variable circle is as easy and rapid as possible. Probably the easiest course would be (without using the conic at all) to calculate numerically, for a given position of the variable sphere, the terrestrial latitude and longitude of the two points of intersection of the variable sphere by the axial circle; laying these down on the projection, we have then a position of the variable circle; and a small number of properly selected positions would give the penumbral curve with tolerable accuracy.

I have throughout spoken of the penumbral curve, as it is in regard hereto that a graphical construction is most needed; but the theory is applicable, without any alteration, to the umbral curve. 